Document downloaded from:

http://hdl.handle.net/10251/162370

This paper must be cited as:

Cuperus, JT.; Carbonell, A.; Fahlgren, N.; Garcia-Ruiz, H.; Burke, RT.; Takeda, A.; Sullivan, CM.... (2010). Unique functionality of 22-nt miRNAs in triggering RDR6-dependent siRNA biogenesis from target transcripts in Arabidopsis. Nature Structural \& Molecular Biology. 17(8):997-1003. https://doi.org/10.1038/nsmb.1866

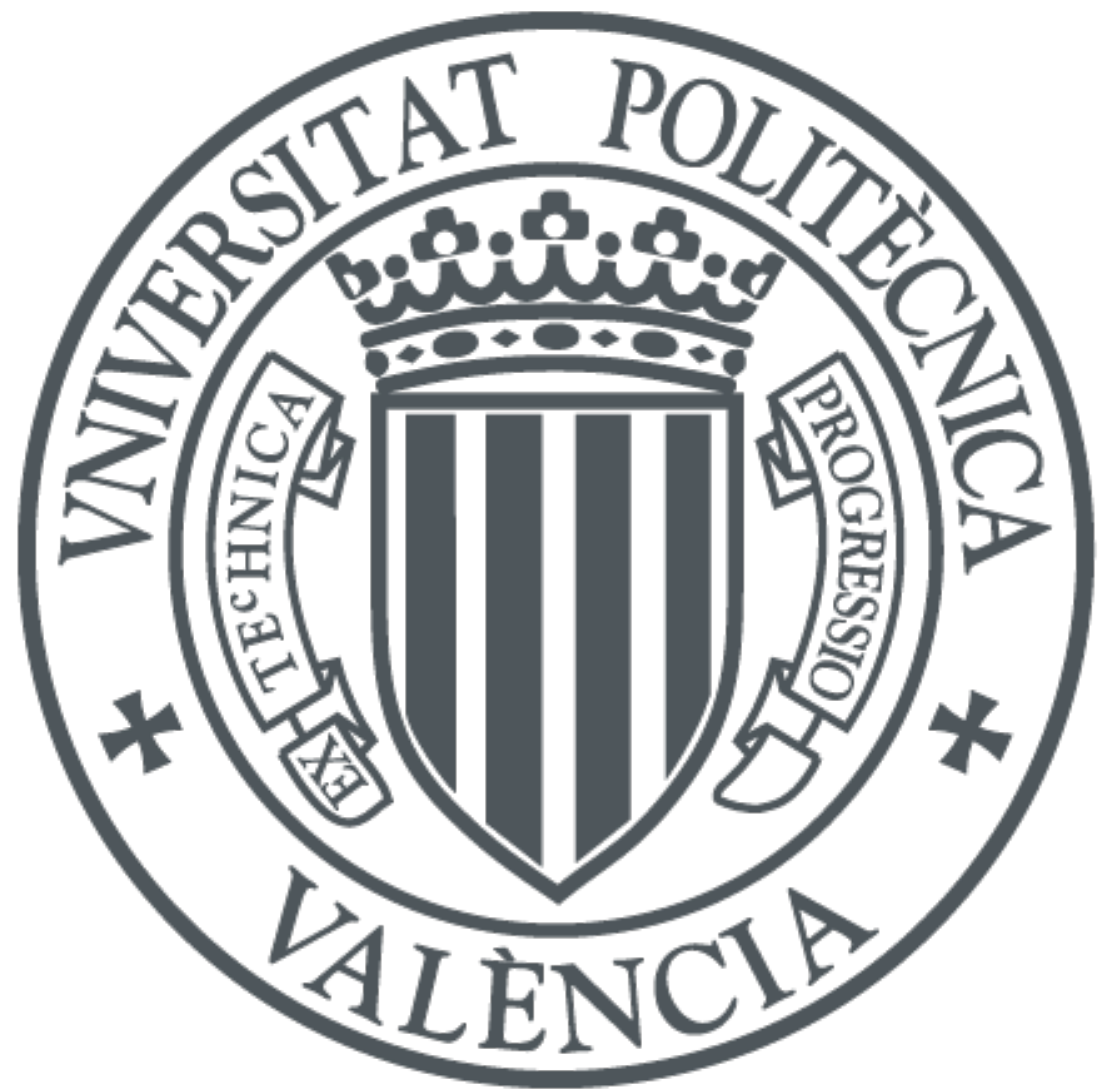

The final publication is available at

https://doi.org/10.1038/nsmb.1866

Copyright Nature Publishing Group

Additional Information 


\section{Unique Functionality of 22 nt miRNAs in Triggering RDR6-Dependent siRNA Biogenesis from Target Transcripts in Arabidopsis}

Josh T. Cuperus ${ }^{1,2,6}$, Alberto Carbonell ${ }^{2,6}$, Noah Fahlgren ${ }^{1,2}$, Hernan Garcia-Ruiz², Russell T. Burke ${ }^{2}$, Atsushi Takeda ${ }^{4}$, Christopher M. Sullivan ${ }^{2,3}$, Sunny D. Gilbert ${ }^{2,3}$, Taiowa A. Montgomery ${ }^{1,5}$ and James C. Carrington ${ }^{2,3}$,

${ }^{1}$ Molecular and Cellular Biology Program, '2Department of Botany and Plant Pathology, and ${ }^{3}$ Center for Genome Research and Biocomputing, Oregon State University, Corvallis, OR 97331

${ }^{4}$ Present address: Department of Life Sciences, Graduate School of Arts and Sciences, The University of Tokyo, Tokyo 153-8902, Japan

${ }^{5}$ Present address: Department of Molecular Biology, Massachusetts General Hospital, 185 Cambridge Street, CPZN7250, Boston, MA 02114, USA

${ }^{6}$ These authors contributed equally to this work Correspondence should be addressed to J.C.C. (carrington@cgrb.oregonstate.edu) 
RNA interference pathways may involve amplification of secondary siRNAs by RNAdependent RNA polymerases. In plants, RDR6-dependent secondary siRNAs arise from transcripts targeted by some microRNA (miRNA). Here, Arabidopsis thaliana secondary siRNA from mRNA, and trans-acting siRNA, are shown to be triggered through initial targeting by $22 \mathrm{nt}$ miRNA that associate with AGO1. In contrast to canonical 21 nt miRNA, 22 nt miRNA primarily arise from foldback precursors containing asymmetric bulges. Using artificial miRNA constructs, conversion of asymmetric foldbacks to symmetric foldbacks resulted in production of $21 \mathrm{nt}$ forms of miR173, miR472 and miR828. Both 21 and $22 \mathrm{nt}$ forms associated with AGO1 and guided accurate slicer activity, but only $22 \mathrm{nt}$ miRNA were competent to trigger RDR6-dependent siRNA from target RNA. These data suggest that AGO1 functions differentially with 21 and 22 nt miRNA to engage the RDR6-associated amplification apparatus. 


\section{INTRODUCTION}

During RNA interference (RNAi), double stranded RNA (dsRNA) is processed by Dicer, a dsRNA-specific RNase III class ribonuclease, into small 20-30 nt RNA duplexes.

Typically, one strand of the duplex preferentially associates with an Argonaute protein to form an effector complex (RNA-induced silencing complex, or RISC), that targets and silences transcripts based on sequence complementarity ${ }^{1}$. In plants, fungi, nematodes, and some other organisms, this process can be amplified through production of secondary short interfering RNAs (siRNAs) after transcription by RNA-dependent RNA polymerase (RdRp) on the primary target RNA ${ }^{2}$. As exemplified in Caenorhabditis elegans, primary siRNA, secondary siRNA and other small RNA may serve as guides in association with distinct Argonaute proteins ${ }^{3-5}$. Additionally, dsRNA precursors or siRNA have the potential to act non-cell-autonomously in plants and some animals, leading to spread of silencing signals, subsequent amplification, and transgenerational silencing in some cases ${ }^{2,6}$.

In amplification-competent organisms, the selective recruitment and activity of RdRp on primary targets is a key step that governs whether or not secondary siRNA and amplification occurs. The partially redundant RdRps RRF-1 and EGO-1 from C. elegans, QDE-1 from Neurospora crassa, Rdp1 from Schizosaccharomyces pombe, and RNADEPENDENT RNA POLYMERASE6 (RDR6) from Arabidopsis thaliana transcribe RNA in a primer-independent manner ${ }^{7-12}$. A. thaliana may encode six RdRps, at least three of which generate long dsRNA that serve as DICER-LIKE (DCL) substrates in several distinct pathways $^{2,13}$. RDR6, which frequently functions in combination with DCL4, participates in posttranscriptional silencing of exogenous targets (transgene transcripts, some viral RNA), as well as several types of endogenous transcripts ${ }^{13,14}$. Indiscriminant entry of transcripts into the RDR6-dependent siRNA amplification pathway does not occur in plants. Amplification of secondary siRNA occurs, however, from a minority of small RNA-targeted transcripts, and the genetic requirements for biogenesis of these siRNA resemble those of 
trans-acting siRNA (tasiRNA) ${ }^{15-20}$. Tightly controlled entry of transcripts into amplification pathways makes intuitive sense, as feed-forward amplification of silencing signals could conceivably result in runaway suppression of off-target transcripts from related gene family members. In Arabidopsis, the XRN family of exonucleases functions to degrade sliced transcript fragments ${ }^{21}$, and sequencing analysis using an $x r n 4$ mutant suggests that XRN4 is antagonistic to secondary siRNA biogenesis ${ }^{22}$. How secondary siRNA-producing transcripts are routed differentially from most other target transcripts is understood poorly.

Plant tasiRNA represent a case in which an RdRp-dependent, secondary siRNAproducing pathway has evolved to produce specialized small RNA that function to repress co-evolved targets. The four known families of tasiRNA (TAS1-TAS4) in Arabidopsis are particularly informative as experimental systems to understand formation of secondary siRNA. tasiRNAs form from primary TAS transcripts that are initially targeted and sliced by AG01-miR173 (TAS1, TAS2), AGO1-miR828 (TAS4) or AGO7-miR390 (TAS3) complexes ${ }^{18,23-26}$. TAS1, TAS2 and TAS4 tasiRNA-generating transcripts are cleaved at a 5'-proximal site. The 3' RNA product is then transcribed by RDR6, leading to dsRNA that is sequentially processed by DCL4 to yield phased tasiRNA in register with the cleavage site $^{18,23,26-30}$.

Here, the basis for selective entry of miRNA-targeted transcripts through the RDR6dependent siRNA-generating pathway was investigated. Combining genome-wide analyses of $21 \mathrm{nt}$ siRNA and miRNA-targeting patterns with directed experiments, miRNA length was found to be a key determinant in triggering amplification in the context of AGO1-miRNA complexes. In addition, determinants within MIRNA foldbacks that govern miRNA size were identified.

\section{RESULTS}

\section{Secondary siRNAs originate from some targeted mRNAs}


Six A. thaliana small RNA libraries were generated from aerial plant tissue and sequenced in a multiplexed format, yielding an average of 658,999 reads/library that mapped to at least one genomic position. Among 20-24 nt reads, 53.9\% were $21 \mathrm{nts}$, the vast majority of which corresponded to miRNA (Supplementary Fig. 1). A significant proportion of 20-24 nt reads $(74.4 \%)$ mapped to 979 annotated transcripts, 55 of which are known to be targeted by one or more miRNA or tasiRNA (Fig. 1a, Supplementary Table 1). Although targeted transcripts represented only $5.6 \%$ of the 979 small RNA-generating transcripts, reads from the targeted set represented $27.0 \%$ of reads from the entire transcript set. Among reads mapping to the targeted transcripts, $85.4 \%$ were $21 \mathrm{nt}$ in length, whereas the majority $(52.9 \%)$ of reads derived from non-targeted transcripts were 24 nts in length (Fig. 1a,b). Twenty-one-nucleotide siRNA originating from the targeted transcripts were shown previously to be largely RDR6- and DCL4-dependent ${ }^{15}$. Thus, among Arabidopsis siRNA originating from annotated transcripts, those from targeted transcripts are more likely to yield siRNA of $21 \mathrm{nts}$ in length.

A total of 280 miRNA-target or tasiRNA-target pairs were collated from published data (Supplementary Table 2). Given that multigene families with many members targeted by a specific miRNA or tasiRNA family tend to bias representation on this list, "nonredundant" sets of miRNA-target and tasiRNA-target pairs were generated. One hundred non-redundant set iterations (50 pairs in each) contained only one paralog/target family/small RNA, with the targeted family member chosen randomly for each family in each iteration. Most targeted transcripts from the non-redundant list yielded no $21 \mathrm{nt}$ siRNA (Fig. 1c, Supplementary Table 2). However, on average 33.8\% of targets yielded at least a few siRNAs, with several targets yielding highly abundant 21 nt siRNA (Fig. 1c). Levels of these abundant siRNAs were decreased in the $d c / 2-1 d c / 3-1 d c / 4-2$ (termed dcl234) triple mutant (Fig. 1c). 
In exploring the differences between small RNA-target pairs that led to secondary siRNA production and those that did not, it was noticed that secondary siRNA-generating transcripts were frequently targeted by miRNA or tasiRNA of 22 nts in length (Fig. 1d). Targets that interacted with miRNA or tasiRNA containing less than $22 \mathrm{nts}$ yielded significantly ( $p=0.034$, permutation test) less siRNA on average. Additionally, $47 \%$ of singly targeted transcripts and $43 \%$ of multiply targeted transcripts presented in Figure 1a were targeted by a small RNA of predominantly 22 nts in length. The biogenesis and activity of $22 \mathrm{nt}$ miRNAs were therefore subjected to further analysis.

\section{Asymmetric MIRNA foldbacks yield 22 nt miRNA}

The vast majority of Arabidopsis miRNA reads were either 21 or 22 nts in length, with 22 nt families representing $22.5 \%$ of all annotated MIRNA families (Fig. 2a). The overall abundance of 22 nt miRNA reads, however, was relatively low (2.3\%). Both the percentage of $22 \mathrm{nt}$-generating MIRNA families, and the numbers of $22 \mathrm{nt}$ miRNA reads, were comparably low in rice (Fig. 2a; Supplementary Table 3,4). Size was also a distinct characteristic for most MIRNA families in both Arabidopsis and rice, with relatively few families expressing a mixed size distribution. Comparing the proportions of 21 or $22 \mathrm{nt}$ miRNA from non-redundant loci in Arabidopsis and rice revealed a distinct bimodal distribution; over $90 \%$ of all MIRNA loci generated miRNA that were $>80 \%$ either 21 nt or $22 \mathrm{nt}$ in length (Fig. 2b; Supplementary Table 3,4). To understand the basis for 21 vs. 22 nt miRNA formation, foldback base-pairing patterns of Arabidopsis MIRNA were examined. Strikingly, those Arabidopsis foldbacks that contained asymmetry in the form of a non-paired nucleotide within the miRNA sequence were significantly enriched for $22 \mathrm{nt}$ miRNA reads ( $p<2.610^{-9}$, Wilcoxon rank sum test) (Fig. 2c). In nearly all cases of predominantly $22 \mathrm{nt}$ miRNA, including miR173, miR393 and miR472, an asymmetric nonpaired base was detected; there were a few exceptions, such as miR828, which arose 
from a foldback containing only symmetric mispairs within the miRNA/miRNA* segment (Fig. 2d, Supplementary Table 3).

To determine if the asymmetric positions within miRNA sequences of foldbacks led to formation of the 22 nt size miRNA, artificial miRNA (amiRNA) were expressed using foldbacks containing either asymmetric (wild-type configuration) or symmetric miRNA/miRNA* segments. These were produced using the MIR390a foldback, in which the $\mathrm{miR} 390 / \mathrm{miR} 390^{*}$ sequences were replaced by $\mathrm{miR} 173 / \mathrm{miR} 173$ * sequences $^{25}$. The symmetric foldback was engineered by the addition of a $U$ residue to base-pair with the normally mispaired A residue within the MIR173 foldback (Fig. 3a). The authentic MIR173 foldback, and the asymmetric and symmetric amiRNA foldbacks, were coexpressed with the tasiRNA-generating construct, 35S:TAS1c, in transient assays using Nicotiana benthamiana $^{23}$, and both miRNA and tasiRNA accumulation (see below) were measured in blot assays and by sequence analysis. Neither miR173 nor TAS1c tasiRNA are conserved in Nicotiana species, so accumulation of these small RNAs is entirely dependent on the transient expression assay. The asymmetric foldback, from either the authentic (35S:MIR173) or artificial (35S:amiR173) constructs, yielded predominantly 22 nt-size miR173 in blot assays (Fig. 3b). In contrast, the symmetric foldback (35S:amiR173-21) yielded a 21 nt-size miR173 (Fig. 3b). Sequence analysis of small RNAs from these assays confirmed that the asymmetric foldbacks yielded predominantly $22 \mathrm{nt}$ miR173, although a substantial amount of off-size products of 19 and $20 \mathrm{nts}$ were sequenced (Fig. 3c, Supplementary Fig. 2, Supplementary Table 5). The symmetric foldback, however, yielded miR173 that was $91 \% 21$ nt in length (Fig. 3c, Supplementary Fig. 2, Supplementary Table 5). The $21 \mathrm{nt}$ form lacked the $3^{\prime} \mathrm{C}$ residue present in authentic miR173.

Several previous reports collectively showed that miR173 formation requires DCL1, but not DCL2 ${ }^{30-32}$. DCL2, however, was shown to produce 22 nt siRNA from endogenous 
Arabidopsis dsRNA ${ }^{27,29}$. To determine if DCL2, or siRNA-generating DCL3 and DCL4, are necessary for 22 nt miRNA, miRNA reads sequenced from Col-0 and the dc/234 triple mutant were compared. Accumulation and family representation levels of most miRNAs in the $d c / 234$ triple mutant, regardless of size, were similar to those in wild-type Col-0 plants (Fig. 2a, Supplementary Fig. 3a,b). miRNA reads from Col-0 and dcl234 mutant plants for most families, except for two (miR822 and miR839, which are known to be DCL4dependent ${ }^{18}$ ), were highly correlated (Pearson product-moment correlation coefficient $[r]=$ 0.95) (Supplementary Fig. 3b). A high correlation ( $r=0.94)$ was shown when only $22 \mathrm{nt}$ miRNA reads were compared (Supplementary Fig. 3b). Therefore, all known $22 \mathrm{nt}$ miRNA in Arabidopsis are likely DCL1-dependent.

\section{$22 \mathrm{nt}$ forms of miR173, miR472, and miR828 trigger siRNAs}

As shown previously ${ }^{23,30}$ and in Figure 3b, coexpression of 35S:MIR173 with 35S:TAS1c triggered tasiRNA biogenesis. Authentic-size miR173 generated from 35S:amiR173 also triggered TAS1c tasiRNA formation (Fig. 3b, lane 5). The tasiRNA pools triggered by both 22 nt miR173 sources were highly phased, with the register set by the miR173 cleavage site (Fig. 3c, Supplementary Fig. 2, Supplementary Table 6). The $21 \mathrm{nt}$ form produced from the amiR173-21 foldback, however, possessed very low tasiRNA trigger activity, and the few TAS1c-derived siRNA that were produced exhibited no phasing pattern (Fig. $\mathbf{3 b}$,

\section{c; Supplementary Fig. 2; Supplementary Table 6).}

The lack of tasiRNA-trigger activity of miR173-21 could be due to one or more reasons, including failure to associate with AGO1, failure to interact with and guide cleavage of the TAS1c primary transcript, or loss of post-cleavage functions necessary to recruit RDR6 to the precursor transcript. Mi et al. showed that, among AGO1, AGO2, AGO4 and AGO5, miR173 associated most commonly with AGO1. To confirm that both 21 and $22 \mathrm{nt}$ forms of miR173 associated with AGO1, TAS1c biogenesis assays in $N$. 
benthamiana were done with co-expression of HA-tagged AGO1, followed by coimmunoprecipitation analysis. Both 22 and 21 nt forms of miR173 co-immunoprecipitated with HA-AGO1 (Fig. 3d, lanes 4, 6 and 8). TAS1c tasiR255, produced in the presence of 22 nt miR173, also co-immunoprecipitated with HA-AGO1 (Fig. 3d, lanes 4 and 6). As HA-AGO1-nonassociated controls, accumulation TAS1c 3'D2(-) tasiRNA, which contains an AGO1-nonpreferred 5'A, and U6 RNA were analyzed. No or very low levels of TAS1C 3'D2(-) tasiRNA and U6 RNA were detected in the immunoprecipitated samples (Fig. 3d), indicating that HA-AGO1 was selective. To assess more broadly whether or not AGO1 associates preferentially with 21 or 22 nt miRNA, small RNAs from total RNA (input fraction) and immunoprecipitated HA-AGO1 complexes (IP fraction) were identified by high-throughput sequencing (two replicates each). Of the miRNAs that were enriched at least 2-fold in the IP fraction, miRNA families that were predominantly 20-24 nts were recovered, suggesting that AGO1 does not include or exclude miRNA based on length (Supplementary Fig. 4). All predominantly $22 \mathrm{nt}$ miRNA families that met read thresholds for inclusion in the enrichment calculation were enriched in the IP fraction. Additionally, for two predominantly $21 \mathrm{nt}$ miRNAs (miR397a and miR167a,b,d) that had a $>30 \%$ subpopulation of $22 \mathrm{nt}$ variants (Fig. 2b), both the 21 and $22 \mathrm{nt}$ variants were enriched more than 2-fold in the IP fraction (Supplementary Fig. 4).

To assess guide function of 22 and $21 \mathrm{nt}$ miR173 forms, 5' RACE assays were done for each co-expression assay using primers to detect the 3' product of miR173-guided cleavage. Products were detected in all three co-expression assays, and sequencing confirmed that cleavage guided by both 22 and $21 \mathrm{nt}$ miR173 forms occurred at the canonical target site (Fig. 3e,f). Furthermore, both 22 and 21 nt miR173 forms destabilized TAS1c full-length transcripts to very low or undetectable levels (Supplementary Fig. 5).

Because the $21 \mathrm{nt}$ form derived from the 35S:amiR173-21 foldback contained a 3'A rather than a $3^{\prime} \mathrm{C}$ residue, as is present in $22 \mathrm{nt}$ authentic miR173, the effect of $3^{\prime}$ 
nucleotide identity on both 21 and 22 nt forms of miR173 was examined. First, a construct (35S:amiR173-21-3'C) that generated a symmetric foldback yielding a $21 \mathrm{nt}$ form containing a 3'C was tested (Supplementary Fig. 5a). This $21 \mathrm{nt}$-size miR173 failed to trigger tasiRNA accumulation despite guiding accurate cleavage of the TAS1c transcript (Supplementary Fig. 5b,c lane 2). Second, to assess the possibility of a preference for a specific 3' nucleotide in a 22 nt context, 22 nt miR173 variants containing 3' G, A or U were tested. Each of the variants accumulated to within $36 \%$ of the level of $22 \mathrm{nt} \mathrm{miR} 173$ containing a natural $3^{\prime} \mathrm{C}$ and guided accurate cleavage of the TAS1C transcript

(Supplementary Fig. 5b,c). Interestingly, although each of the 22 nt 3'G, 3'A and 3'U variants triggered tasiRNA accumulation, the levels of tasiRNA generated were relatively low (Supplementary Fig. 5b).

To determine if the $22 \mathrm{nt}$ requirement to trigger tasiRNA is specific to miR173, 22 and $21 \mathrm{nt}$ artificial miRNAs for both miR472 and miR828 were generated using the MIR390abased foldback, and tested using the TAS1c tasiRNA transient assay system. Both asymmetric and symmetric foldbacks were engineered following the same strategy as for the miR173 variants (Fig. 4a) and co-expressed with modified TAS1C constructs (35S:TAS1C-472 and 35S:TAS1c-828) in which the normal miR173 target site was substituted with sites recognized by miR472 or miR828. Based on mobility of miR472 and miR828 forms in blot assays, 22 and $21 \mathrm{nt}$ variants were generated as predicted from asymmetric and symmetric foldbacks, respectively, although the asymmetric miR828 foldback yielded a mixture of both size classes (Fig. 4b). Importantly, tasiRNA were detected only in those samples containing 22 nt variants of miR472 and miR828 (Fig. 4b). Like the miR173 size variants, both the 22 and $21 \mathrm{nt}$ forms of miR472 and miR828 guided accurate cleavage of the engineered tasiRNA transcripts (Fig. $\mathbf{4 b , c}$ ) and coimmunoprecipitated with HA-AGO1 (Fig. 4d). These data clearly show that $22 \mathrm{nt}$ forms of 
three Arabidopsis miRNAs possess unique functionality to direct tasiRNA or secondary siRNA biogenesis.

\section{DISCUSSION}

This study associated a unique class of Arabidopsis miRNA - those that are 22 nts in length - with triggering formation of secondary, RDR6/DCL4-dependent 21 nt siRNAs from primary miRNA targets. While both $22 \mathrm{nt}$ and $21 \mathrm{nt}$ forms of miR173, miR472 and miR828 associated with AGO1 and guided accurate cleavage of target transcripts, advancement of target RNA fragments through the 21 nt siRNA biogenesis pathway was a unique property of the 22 nt miRNA-target RNA pairs. Previous studies provided hints that $22 \mathrm{nt}$ miRNAs might be associated with secondary siRNA. Arabidopsis MIR168b was shown to generate both 21 and 22 nt forms of miR168 ${ }^{33}$, which targets the transcript encoding AGO1 and leads to secondary siRNAs in a homeostatic regulatory loop ${ }^{33,34}$. In rice, $22 \mathrm{nt}$ miRNA target sites were identified in several abundant siRNA-generating transcripts ${ }^{35}$, suggesting that the 22 nt property may be conserved in angiosperms.

The fact that most plant miRNAs, particularly those from the most highly expressed families, are $21 \mathrm{nt}$ in length may explain why secondary siRNA from most targeted transcripts are absent or in low abundance. While the tasiRNA represent highly refined examples of functional, discrete secondary siRNAs from a non-coding RNA, it is interesting to consider potential functions for the cases in which abundant siRNAs originate from protein-coding transcripts. In at least some examples, secondary siRNA might specifically target transcripts from related family members. For gene families that are evolving or expanding rapidly, secondary targeting may suppress dosage effects. Evidence for the functionality of secondary siRNA from large multigene family transcripts has been presented ${ }^{15,36,37}$. 
It is proposed that $22 \mathrm{nt}$ miRNA-AGO1 complexes, but not $21 \mathrm{nt}$ miRNA-AGO1 complexes, mark transcripts for RDR6-dependent siRNA formation. This could involve AGO1 adopting one of two states - an amplification-trigger state and a non-trigger state. Both states would be competent to interact with and slice targets containing a suitable target site, but only the trigger state would recruit RDR6 to the de-capped (sliced) target. Previous results from experiments with a non-cleavable target of miR173 suggested that secondary siRNA formation depends on a functional cleavage site ${ }^{30}$. Several studies have also shown that all known tasiRNA, and many other RDR6-dependent siRNA in Arabidopsis ${ }^{15,18,19,23}$, originate preferentially adjacent to target cleavage sites. Recruitment of RDR6, therefore, may depend on target cleavage.

Conformational changes of well-characterized Argonaute proteins are known to occur upon interaction with target RNA and release of the guide $3^{\prime}$ end from the PAZ domain ${ }^{38,39}$. How a 22 nt RNA would effect an AGO1 conformation distinct from a 21 nt RNA is not immediately obvious. The proposed trigger state, however, could involve a direct interaction with RDR6 or associated factors, such as SGS3 ${ }^{16,26}$. To date, there are no data suggesting direct interaction between AGO1 and RDR6 or known RDR6-associated factors. Alternatively, conformationally distinct AGO1-small RNA complexes may interact differentially with GW domain proteins, which interact directly with AGO proteins as scaffolds to mediate interactions with other factors to suppress translation and promote de-adenylation ${ }^{40}$. Arabidopsis AGO1-specific GW proteins are not yet characterized, although AGO4 likely interacts with GW domains in an RNA PoIV subunit (NRPDE1) and the transcription elongation factor SPT5 $\mathrm{L}^{41-43}$.

While it is clear that AGO1 exhibits a preference for small RNAs with a 5'U, which docks within a MID domain binding pocket, there are no clear structural or functional data supporting a 3' nucleotide preference for interaction with the PAZ domain. Among $22 \mathrm{nt}$ miRNAs in Arabidopsis, a $3^{\prime} \mathrm{C}$ is most common, although this implies neither a binding 
preference nor particular functionality. Within a $22 \mathrm{nt}$ context, a 3 'C promoted siRNAtrigger function of miR173 most effectively. However, there was no strict requirement for any specific 3' nucleotide, and the $22 \mathrm{nt}$ siRNA-triggers miR828 and miR393 both contain 3'A. The studies presented here do not allow quantitative comparison of the affinity of AGO1 for 21 vs. $22 \mathrm{nt}$ miRNAs, or for $22 \mathrm{nt}$ miRNAs with different 3' nucleotides.

The finding that 22 nt miRNA trigger RDR6-dependent secondary siRNAs leads to the question of whether or not other siRNA amplification pathways have guide RNA-specific activities to recruit RDR1 or RDR2. Signals for amplification mediated by RDR1 and RDR6 during antiviral RNAi are not known, although results presented here suggest $22 \mathrm{nt}$ siRNA, working through AGO1 or other AGO proteins, might be plausible triggers. As DCL2 is known to both promote a full silencing response against several viruses ${ }^{32,44-47}$ and to generate $22 \mathrm{nt}$ siRNAs ${ }^{27,29}$, this idea may have support. DCL2 was also shown to promote DCL4-dependent posttranscriptional silencing of several transgenes ${ }^{48}$. Furthermore, overproduction of DCL2-dependent 22 nt siRNA may account for the severe phenotypes of dc/134 and dc/14 mutant Arabidopsis plants, in which DCL2 might generate 22 nt siRNA from dsRNA that would normally be processed to $21 \mathrm{nt} \mathrm{siRNA}$ by DCL4 ${ }^{32}$.

Finally, the basis for production of either a $21 \mathrm{nt}$ or a $22 \mathrm{nt}$ miRNA depends on the nature of the foldback in plants. Most Arabidopsis miRNAs are formed by DCL1-mediated processing first at the loop-distal end of the foldback, then at the loop proximal end ${ }^{49-51}$. DCL1, like other Dicers, functions as a molecular ruler. For the loop-proximal cuts, DCL1 "measures" the length of helical RNA from the PAZ domain-bound end to the positions juxtaposed to the active centers ${ }^{52-54}$. For foldbacks that are symmetrically paired within the miRNA/miRNA* region, it is inferred that DCL1 measures a length equivalent to 21 base pairs in most cases. However, it is also inferred that length of an A-form helix containing a single asymmetric bulge is frequently equivalent to that of a symmetrically paired helix. Three-dimensional modeling of $21 \mathrm{nt}$ - and $22 \mathrm{nt}$-generating MIR173 foldbacks using MC- 
Fold and MC-Sym ${ }^{55}$ supports this idea. The most likely models show that an unpaired nucleotide does not substantially increase the length of the miRNA/miRNA* duplex region (Supplementary Fig. 6). This explains why, when the asymmetric nucleotide occurs within the miRNA sequence, the resulting miRNA contains a non-canonical $22 \mathrm{nt}$ length. Given the unique functionality of $22 \mathrm{nt}$ miRNA to trigger siRNA amplification, it seems reasonable to propose that foldback structures with asymmetric positions, and that would result in 22 nt miRNA, should be under evolutionary constraints.

Note: Supplementary information is available on the Nature Structural \& Molecular Biology website.

Accession codes. High-throughput sequencing datasets for HA-AGO1-associated small RNA and input controls were deposited in Gene Expression Omnibus (http://www.ncbi.nlm.nih.gov/geo) under the series accession GSE22252. Datasets for Col-0 and dcl2-1 dcl3-1 dcl4-2 small RNA libraries were published previously ${ }^{47}$ and are available under the series accession GSE20197. Twenty-eight O. sativa Nipponbare libraries, as described in Supplementary Information, were also used.

\section{ACKNOWLEDGMENTS}

A.C. was supported by a postdoctoral fellowship from the Ministerio de Ciencia e Innovacion (BMC-2008-0188). This work was supported by grants from the National Science Foundation (MCB-0618433 and MCB-0956526), National Institutes of Health (Al43288) and Monsanto Corporation.

\section{AUTHOR CONTRIBUTIONS}

J.T.C., A.C., N.F., and J.C.C. designed the experiments; J.T.C., A.C., N.F., H.G.R., R.T.B., 
A.T., C.M.S., S.D.G. and T.A.M. performed experiments; J.T.C., A.C., N.F. S.D.G., T.A.M. and J.C.C. analyzed data; J.T.C., A.C., N.F., and J.C.C. wrote the paper.

\section{FIGURE LEGENDS}

Figure 1 Small RNA from Arabidopsis annotated transcripts. (a) Proportion of small RNAgenerating transcripts that are targeted (at single or multiple sites) by miRNA or tasiRNA, or non-targeted. Outer rings show proportion of small RNA size. (b) Mean 20-24 nt siRNA levels from targeted or non-targeted transcripts. (c) Non-redundant small RNA-target transcript pairs yielding four levels (bins) of 21 nt siRNA in Col-0 and dc/2-1 dc/3-1 dcl4-2 (dcl234) mutant plants. Data are from averages of six (Col-0) and five (dcl234) replicates. (d) Box plots showing the mean numbers of $21 \mathrm{nt}$ siRNA originating from non-redundant transcripts targeted by small RNA that are 22 nt or less than $22 \mathrm{nt}$ in length.

Figure 2 MIRNA foldback asymmetry leads to formation of 22 nt miRNAs. (a) Mean proportions of distinct miRNA size classes in read datasets (top), and of predominant size class for MIRNA families (bottom), from Arabidopsis and rice. The rice miRNA were from a filtered subset that passed basic criteria for bona fide miRNA $^{56}$ (see Supplemental Table 4). (b) Rank order showing proportion of 22 nt size class, from averages of sequencing datasets, corresponding to non-redundant MIRNA loci in Arabidopsis and rice. Multigene MIRNA families with loci encoding the identical miRNA, but that have both symmetric and asymmetric foldbacks, are color coded grey. (c) Proportion of 22 nt miRNA from nonredundant MIRNA loci with base-pair asymmetry or symmetry within the miRNA/miRNA* segment of the foldback. (d) Examples of 22 nt miRNA-generating MIRNA foldbacks, average miRNA size distribution, and proportion of target transcripts that yield $21 \mathrm{nt}$ siRNA (at least four reads from 4/6 replicate libraries). Green arrows indicate the predicted 
asymmetric position within the foldbacks.

Figure 3 Production and activities of 21 and 22 nt miR173 forms. (a) Foldbacks of from wild-type MIR173, amiR173 and amiR173-21. Artificial miRNAs were engineered within the MIR390a foldback. miRNA guide and miRNA* strands are represented with green and red, respectively. The arrows indicate the predicted asymmetric position in MIR173 and amiR173 foldbacks. (b) Accumulation of miR173 and TAS1c tasiRNA (siR255) in N. benthamiana transient assays. Constructs were coexpressed as indicated above the blot panels. Mean $(\mathrm{n}=3$ ) relative miR173 (red) and siR255 (blue) levels +/- SD (lane 2 and lane $3=1.0$ for miR173 and tasiRNA255 respectively) were plotted (top). One of three biological replicates of the blot data, and EtBr-stained rRNA as loading controls, are shown (bottom). (c) Analysis of miR173 (from 35S:MIR173, 35S:amiR173 and 35S:amiR173-21) and TAS1c-derived siRNA sequences by high-throughput sequencing after transient assays in $N$. benthamiana. Pie charts display the percentage of $18-24 \mathrm{nt}$ reads. Radar plots display percentages of $21 \mathrm{nt}$ reads corresponding to each of the 21 registers from TAS1c transcripts, with position 1 designated as immediately after the miR173-guided cleavage site. (d) Analysis of co-immunoprecipitation of $21 \mathrm{nt}$ and $22 \mathrm{nt}$ miR173 variants with HA-AGO1. Protein and RNA blot assays using input (in) and IP (HA) fractions from $N$. benthamiana following coexpression of 35S:HA-AGO1 and 35S:TAS1C with 35S:MIR173, 35S:amiR173 and 35S:amiR173-21. The TAS1c 3'D2(-) panel shows an HA-AGO1-nonassociated tasiRNA generated from the TAS1c transcript as an IP control. U6 RNA and EtBr-stained rRNA were included as input loading and HA-AGO1-nonassociated controls. (e) EtBr-stained 5' RACE products corresponding to the 3' cleavage product from miR173-guided cleavage. $N$. benthamiana actin RT-PCR products are shown as a control. (f) Proportion of cloned 5' RACE products corresponding to cleavage within TAS1c transcripts at the canonical miR173-guided site in assays with amiR173 and 
amiR173-21.

Figure 4 Production and activities of 21 and $22 \mathrm{nt}$ miR472 and miR828 forms. (a) Foldbacks of amiR828, amiR828-21, amiR472, and amiR472-21. (b) Accumulation of miR472, miR828 and modified TAS1c tasiRNA (siR255), and 5'RACE to detect miRNAguided cleavage products of the modified TAS1c transcripts, in N. benthamiana transient assays. (c) Proportion of cloned 5' RACE products corresponding to cleavage within modified TAS1c transcripts at the canonical miR472- or miR828-guided sites in assays with the designated artificial miRNAs. The target site sequences are actual sites from At1g12290 and TAS4 transcripts, which are recognized by miR472 and miR828, respectively. (d) Analysis of co-immunoprecipitation of $21 \mathrm{nt}$ and $22 \mathrm{nt}$ amiR472 and amiR828 variants with HA-AGO1. Protein and RNA assays for input (in) and IP (HA) fractions from $N$. benthamiana expressing the amiR472 and amiR828 variants were done using blots containing samples from both sets of experiments. U6 RNA and EtBr-stained rRNA were included as input loading and HA-AGO1-non-associated controls.

\section{METHODS}

Plant materials and growth conditions. N. benthamiana and A. thaliana plants, including the previously described $d c / 2-1 d c / 3-1 d c / 4-2$ triple mutant ${ }^{47}$, were grown in normal greenhouse conditions with supplemental light on a 16hr light/ $8 \mathrm{hr}$ dark cycle.

Transgene constructs. 35S:TAS1C and 35S:MIR173a derived constructs were generated in pMDC32 and described previously 23,25,30,57. MIR390a-derived artificial miRNA constructs were designed by ligating overlapping oligonucleotides into a pMDC32-derived vector containing $~ 200 \mathrm{bp}$ upstream and downstream of the MIR390a foldback. 
Transient expression assays. Transient expression assays in $N$. benthamiana leaves were carried out as previously described ${ }^{58}$ with Agrobacterium tumefaciens GV3101 . Bacterial cultures were resuspended at $\mathrm{OD}_{600}=1.0$. When multiple constructs were coexpressed, equal amounts of each culture were used. Plants were grown in the greenhouse after infiltration, and zones of infiltration were harvested 48 hours postinfiltration for RNA isolation.

RNA Blot assays. Total RNA from Arabidopsis and N. benthamiana was isolated using TRIzol reagent (Invitrogen). Two chloroform extractions were done and RNA was precipitated in an equal volume of isopropanol for $20 \mathrm{~min}$. Triplicate samples from pools of N. benthamiana infiltrated leaves were analyzed. RNA blot assays were done as described $^{30}$. Briefly $5 \mu \mathrm{g}, 10 \mu \mathrm{g}$ or $20 \mu \mathrm{g}$ of total normalized RNA was resolved by denaturing polyacrylamide-gel electrophoresis for small RNA analysis, or $5 \mu \mathrm{g}$ of total RNA was resolved by denaturing $1.5 \%$ agarose-gel electrophoresis. RNA was transferred to positively charged nitrocellulose membrane. DNA or LNA probes were end-labeled using $\left.{ }^{[2}\right]$ ATP and Optikinase (USB). Probes were hybridized to RNA on membranes in Sigma Perfect-Hyb at $68^{\circ} \mathrm{C}$ (HMW blots) or $38-42^{\circ} \mathrm{C}$ (small RNA blots). An Instant Imager (Packard Bioscience) was used to measure blot hybridization signals.

Small RNA sequencing. Small RNA amplicons from transient assays in $N$. benthamiana were prepared as described ${ }^{57,59}$. Four synthetic oligoribonucleotides were added in different amounts (Std11, $0.0001 \mathrm{pmol}$; Std6, $0.001 \mathrm{pmol}$; Std3, 0.01 pmol; Std2, $0.1 \mathrm{pmol}$ ) to $90 \mu \mathrm{g}$ total RNA per sample before amplicon preparation. Sequencing-by-synthesis was done with five pmol of each amplicon using an Illumina Genome Analyzer II (GAll, www.illumina.com). Reads were parsed using the first six nucleotides of the 3' adapter (CTGTAG). Reads were normalized per million as described ${ }^{59}$. Read proportions were 
based on total reads (18-24 nts) that matched perfectly within a 29 base window surrounding the miR173 or miR173* sequences from samples containing 35S:MIR173a, 35S:amiR173 and 35S:amiR173-21. Radar plots to display phasing from 35S:TAS1c were generated as previously described ${ }^{19,25}$. Small RNA amplicons were generated and analyzed from HA-AGO1 IP samples as described ${ }^{25}$.

Small RNA analysis. Small RNA from A. thaliana were mapped to the genome and annotated transcriptome (TAIR9; http://www.arabidopsis.org), including annotated MIRNA precursors (miRBase v14; http://www.miRBase.org), using the CASHX pipeline ${ }^{59}$. Those transcripts to which four or greater small RNA reads/million (RPM) were mapped in at least four of the six libraries were defined as small RNA-generating transcripts. For analysis of small RNA-generating transcripts, annotated structural RNA, pseudogenes or transposable elements were removed.

Statistical analyses. All statistical analyses were done using R v2.9.2. Permutation tests were done using the $\mathrm{R}$ "twotPermutation" function, with 1 million simulations, from the DAAG package ${ }^{60}$. A Wilcoxon rank sum test was used to compare empirical logits for the ratio of 22 to $21 \mathrm{nt}$ miRNA reads. The rank sum test was done using the $\mathrm{R}$ "wilcox.test" function from the stats package.

5' RACE. Modified RNA ligase-mediated 5' RACE was done as described ${ }^{30}$ with the Generacer kit (Invitrogen) using the following gene specific primers:

TAS1C_707_5'_RACE [GATGATGCTTCTTCGCTACACCTCGGAG] and TAS1c_573_5'_RACE [AGCAACTGTTCTTTAGACGACTTGAAAATCTCAT]. 5' RACE products were gel purified using Invitrogen PureLink gel extraction kit, cloned in TOPO TA (Invitrogen), introduced into E. coli, screened for inserts, and sequenced. 


\section{REFERENCES}

1. Kim, V.N., Han, J. \& Siomi, M.C. Biogenesis of small RNAs in animals. Nat Rev Mol Cell Biol 10, 126-139 (2009).

2. Ghildiyal, M. \& Zamore, P.D. Small silencing RNAs: an expanding universe. Nat Rev Genet 10, 94-108 (2009).

3. Guang, S. et al. An Argonaute transports siRNAs from the cytoplasm to the nucleus. Science 321, 537-541 (2008).

4. Gu, W. et al. Distinct argonaute-mediated 22G-RNA pathways direct genome surveillance in the C. elegans germline. Mol Cell 36, 231-244 (2009).

5. Claycomb, J.M. et al. The Argonaute CSR-1 and its 22G-RNA cofactors are required for holocentric chromosome segregation. Cell 139, 123-134 (2009).

6. Carthew, R.W. \& Sontheimer, E.J. Origins and Mechanisms of miRNAs and siRNAs. Cell 136, 642-655 (2009).

7. Meister, G. \& Tuschl, T. Mechanisms of gene silencing by double-stranded RNA. Nature 431, 343-349 (2004).

8. Makeyev, E.V. \& Bamford, D.H. Cellular RNA-dependent RNA polymerase involved in posttranscriptional gene silencing has two distinct activity modes. $\mathrm{Mol}$ Cell 10, 1417-1427 (2002).

9. Sijen, T., Steiner, F.A., Thijssen, K.L. \& Plasterk, R.H. Secondary siRNAs result from unprimed RNA synthesis and form a distinct class. Science 315, 244-247 (2007).

10. Pak, J. \& Fire, A. Distinct populations of primary and secondary effectors during RNAi in C. elegans. Science 315, 241-244 (2007).

11. Motamedi, M.R. et al. Two RNAi complexes, RITS and RDRC, physically interact and localize to noncoding centromeric RNAs. Cell 119, 789-802 (2004).

12. Correa, R.L., Steiner, F.A., Berezikov, E. \& Ketting, R.F. MicroRNA-directed siRNA biogenesis in Caenorhabditis elegans. PLoS Genet 6, e1000903 (2010).

13. Voinnet, $O$. Use, tolerance and avoidance of amplified RNA silencing by plants. Trends Plant Sci 13, 317-328 (2008).

14. Mallory, A.C., Elmayan, T. \& Vaucheret, H. MicroRNA maturation and action--the expanding roles of ARGONAUTEs. Curr Opin Plant Biol 11, 560-566 (2008).

15. Howell, M.D. et al. Genome-wide analysis of the RNA-DEPENDENT RNA POLYMERASE6/DICER-LIKE4 pathway in Arabidopsis reveals dependency on miRNA- and tasiRNA-directed targeting. Plant Cell 19, 926-942 (2007). 
16. Peragine, A., Yoshikawa, M., Wu, G., Albrecht, H.L. \& Poethig, R.S. SGS3 and SGS2/SDE1/RDR6 are required for juvenile development and the production of trans-acting siRNAs in Arabidopsis. Genes Dev 18, 2368-2379 (2004).

17. Vazquez, F. et al. Endogenous trans-acting siRNAs regulate the accumulation of Arabidopsis mRNAs. Mol Cell 16, 69-79 (2004).

18. Rajagopalan, R., Vaucheret, H., Trejo, J. \& Bartel, D.P. A diverse and evolutionarily fluid set of microRNAs in Arabidopsis thaliana. Genes Dev 20, 3407-3425 (2006).

19. Axtell, M.J., Jan, C., Rajagopalan, R. \& Bartel, D.P. A Two-Hit Trigger for siRNA Biogenesis in Plants. Cell 127, 565-577 (2006).

20. Ronemus, M., Vaughn, M.W. \& Martienssen, R.A. MicroRNA-targeted and small interfering RNA-mediated mRNA degradation is regulated by argonaute, dicer, and RNA-dependent RNA polymerase in Arabidopsis. Plant Cell 18, 1559-1574 (2006).

21. Chiba, Y. \& Green, P.J. mRNA degredation machinery in plants. J. Plant Biol. 52, 114-124 (2009).

22. Gregory, B.D. et al. A link between RNA metabolism and silencing affecting Arabidopsis development. Dev Cell 14, 854-866 (2008).

23. Allen, E., Xie, Z., Gustafson, A.M. \& Carrington, J.C. microRNA-directed phasing during trans-acting siRNA biogenesis in plants. Cell 121, 207-221 (2005).

24. Baumberger, N. \& Baulcombe, D.C. Arabidopsis ARGONAUTE1 is an RNA slicer that selectively recruits microRNAs and short interfering RNAs. Proc Natl Acad Sci USA 102, 11928-11933 (2005).

25. Montgomery, T.A. et al. Specificity of ARGONAUTE7-miR390 interaction and dual functionality in TAS3 trans-acting siRNA formation. Cell 133, 128-141 (2008).

26. Yoshikawa, M., Peragine, A., Park, M.Y. \& Poethig, R.S. A pathway for the biogenesis of trans-acting siRNAs in Arabidopsis. Genes Dev 19, 2164-2175 (2005).

27. Xie, Z., Allen, E., Wilken, A. \& Carrington, J.C. DICER-LIKE 4 functions in transacting small interfering RNA biogenesis and vegetative phase change in Arabidopsis thaliana. Proc Natl Acad Sci USA 102, 12984-12899 (2005).

28. Dunoyer, P., Himber, C. \& Voinnet, O. DICER-LIKE 4 is required for RNA interference and produces the 21-nucleotide small interfering RNA component of the plant cell-to-cell silencing signal. Nat Genet 37, 1356-1360 (2005).

29. Gasciolli, V., Mallory, A.C., Bartel, D.P. \& Vaucheret, H. Partially redundant functions of Arabidopsis DICER-like enzymes and a role for DCL4 in producing trans-acting siRNAs. Curr Biol 15, 1494-1500 (2005). 
30. Montgomery, T.A. et al. AGO1-miR173 complex initiates phased siRNA formation in plants. Proc Natl Acad Sci U S A 105, 20055-20062 (2008).

31. Park, W., Li, J., Song, R., Messing, J. \& Chen, X. CARPEL FACTORY, a Dicer homolog, and HEN1, a novel protein, act in microRNA metabolism in Arabidopsis thaliana. Curr Biol 12, 1484-1495 (2002).

32. Bouche, N., Lauressergues, D., Gasciolli, V. \& Vaucheret, H. An antagonistic function for Arabidopsis DCL2 in development and a new function for DCL4 in generating viral siRNAs. EMBO J 25, 3347-3356 (2006).

33. Vaucheret, H. AGO1 homeostasis involves differential production of 21 -nt and 22-nt miR168 species by MIR168a and MIR168b. PLoS One 4, e6442 (2009).

34. Mallory, A.C. \& Vaucheret, H. ARGONAUTE 1 homeostasis invokes the coordinate action of the microRNA and siRNA pathways. EMBO Rep 10, 521526 (2009).

35. Johnson, C. et al. Clusters and superclusters of phased small RNAs in the developing inflorescence of rice. Genome Res 19, 1429-1440 (2009).

36. Chen, H.M., Li, Y.H. \& Wu, S.H. Bioinformatic prediction and experimental validation of a microRNA-directed tandem trans-acting siRNA cascade in Arabidopsis. Proc Natl Acad Sci USA 104, 3318-3323 (2007).

37. Addo-Quaye, C., Eshoo, T.W., Bartel, D.P. \& Axtell, M.J. Endogenous siRNA and miRNA targets identified by sequencing of the Arabidopsis degradome. Curr Biol 18, 758-762 (2008).

38. Wang, Y. et al. Structure of an argonaute silencing complex with a seedcontaining guide DNA and target RNA duplex. Nature 456, 921-926 (2008).

39. Wang, Y. et al. Nucleation, propagation and cleavage of target RNAs in Ago silencing complexes. Nature 461, 754-761 (2009).

40. Eulalio, A., Tritschler, F. \& Izaurralde, E. The GW182 protein family in animal cells: new insights into domains required for miRNA-mediated gene silencing. RNA 15, 1433-1442 (2009).

41. El-Shami, M. et al. Reiterated WG/GW motifs form functionally and evolutionarily conserved ARGONAUTE-binding platforms in RNAi-related components. Genes Dev 21, 2539-2544 (2007).

42. Bies-Etheve, N. et al. RNA-directed DNA methylation requires an AGO4interacting member of the SPT5 elongation factor family. EMBO Rep 10, 649-654 (2009).

43. He, X.J. et al. An effector of RNA-directed DNA methylation in arabidopsis is an ARGONAUTE 4- and RNA-binding protein. Cell 137, 498-508 (2009). 
44. Blevins, T. et al. Four plant Dicers mediate viral small RNA biogenesis and DNA virus induced silencing. Nucleic Acids Res 34, 6233-6246 (2006).

45. Deleris, A. et al. Hierarchical action and inhibition of plant Dicer-like proteins in antiviral defense. Science 313, 68-71 (2006).

46. Diaz-Pendon, J.A., Li, F., Li, W.X. \& Ding, S.W. Suppression of antiviral silencing by cucumber mosaic virus $2 b$ protein in Arabidopsis is associated with drastically reduced accumulation of three classes of viral small interfering RNAs. Plant Cell 19, 2053-2063 (2007).

47. Garcia-Ruiz, H. et al. Arabidopsis RNA-Dependent RNA Polymerases and DicerLike Proteins in Antiviral Defense and Small Interfering RNA Biogenesis during Turnip Mosaic Virus Infection. Plant Cell 22, 481-496 (2010).

48. Mlotshwa, S. et al. DICER-LIKE2 plays a primary role in transitive silencing of transgenes in Arabidopsis. PLoS One 3, e1755 (2008).

49. Mateos, J.L., Bologna, N.G., Chorostecki, U. \& Palatnik, J.F. Identification of MicroRNA processing determinants by random mutagenesis of Arabidopsis MIR172a precursor. Curr Biol 20, 49-54 (2010).

50. Song, L., Axtell, M.J. \& Fedoroff, N.V. RNA secondary structural determinants of miRNA precursor processing in Arabidopsis. Curr Biol 20, 37-41 (2010).

51. Werner, S., Wollmann, H., Schneeberger, K. \& Weigel, D. Structure determinants for accurate processing of miR172a in Arabidopsis thaliana. Curr Biol 20, 42-48 (2010).

52. Macrae, I.J. et al. Structural basis for double-stranded RNA processing by Dicer. Science 311, 195-198 (2006).

53. MacRae, I.J., Zhou, K. \& Doudna, J.A. Structural determinants of RNA recognition and cleavage by Dicer. Nat Struct Mol Biol 14, 934-940 (2007).

54. Qin, H. et al. Structure of the Arabidopsis thaliana DCL4 DUF283 domain reveals a noncanonical double-stranded RNA-binding fold for protein-protein interaction. RNA 16, 474-481 (2010).

55. Parisien, M. \& Major, F. The MC-Fold and MC-Sym pipeline infers RNA structure from sequence data. Nature 452, 51-55 (2008).

56. Meyers, B.C. et al. Criteria for Annotation of Plant MicroRNAs. Plant Cell 20, 3186-3190 (2008).

57. Cuperus, J.T. et al. Identification of MIR390a precursor processing-defective mutants in Arabidopsis by direct genome sequencing. Proc Natl Acad Sci U S A 107, 466-471 (2010). 
58. Llave, C., Xie, Z., Kasschau, K.D. \& Carrington, J.C. Cleavage of Scarecrow-like mRNA targets directed by a class of Arabidopsis miRNA. Science 297, 20532056 (2002).

59. Fahlgren, N. et al. Computational and analytical framework for small RNA profiling by high-throughput sequencing. RNA 15, 992-1002 (2009).

60. Maindonald, J.H. \& Braun, J. Data analysis and graphics using $R$ : an examplebased approach, 502 p. (Cambridge University Press, Cambridge ; New York, 2007). 
a

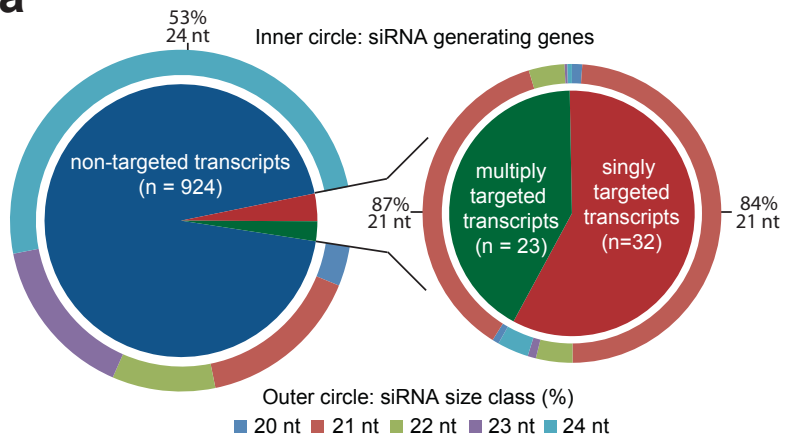

C

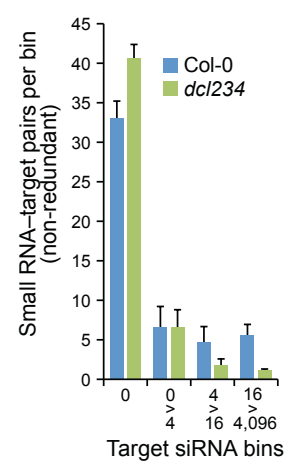

d

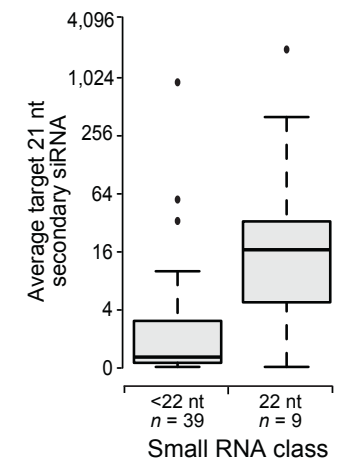

b

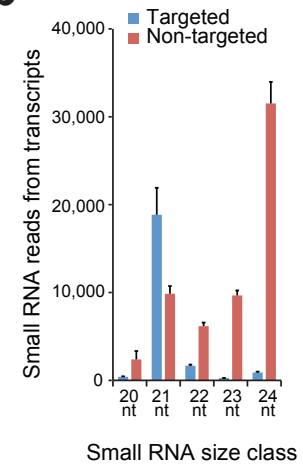

Figure 1 


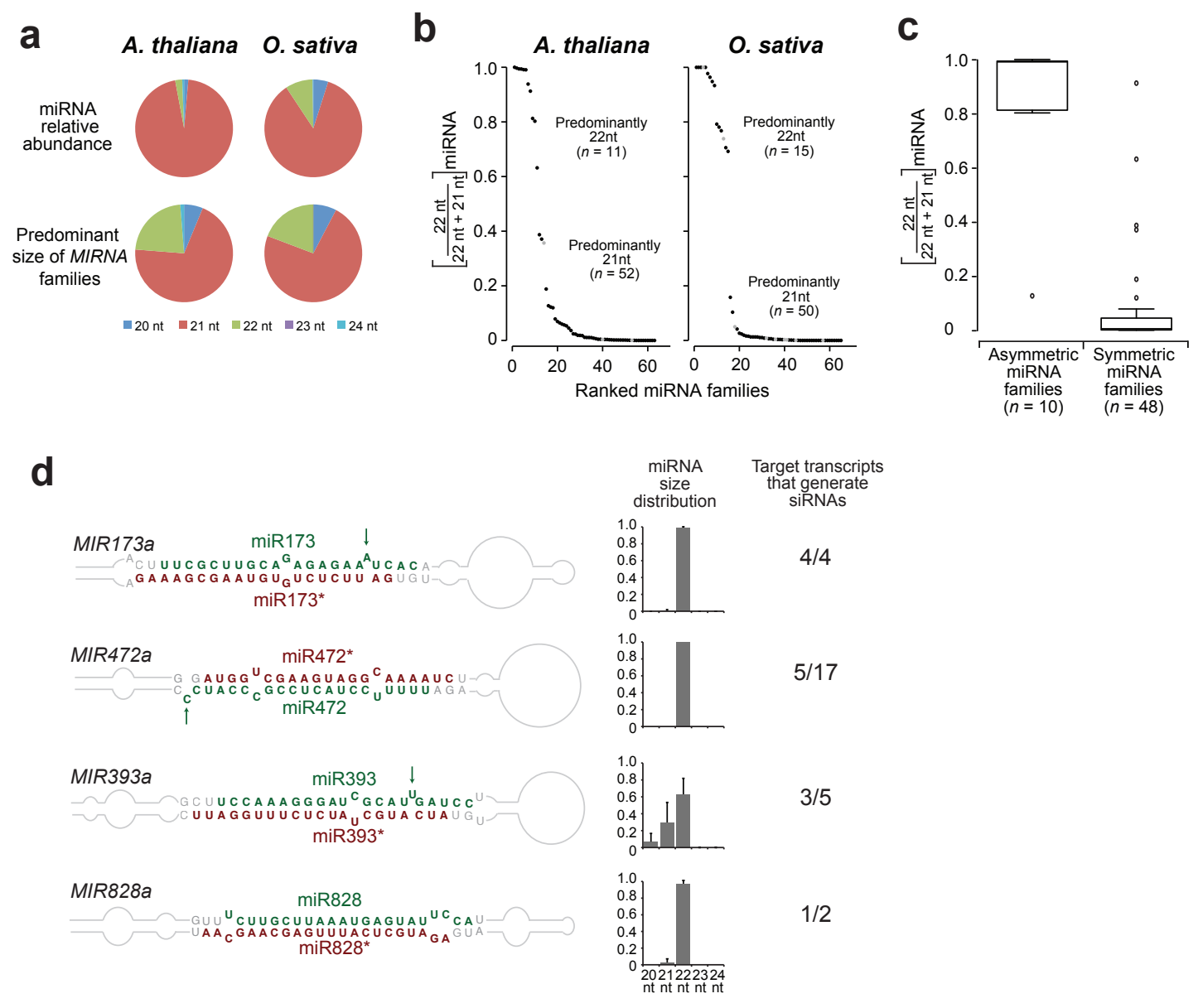

Figure 2 


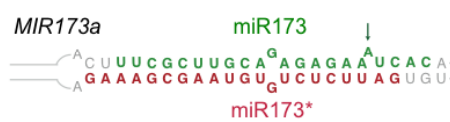

amiR173

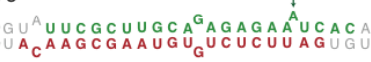

amiR173-21

$G U^{A} \cup \cup C G C U \cup G C A G A G A G A A A U C A C$,
$\cup A C A A G C G A A \cup G U G \cup C \cup C \cup \cup \cup A G \cup G$

b

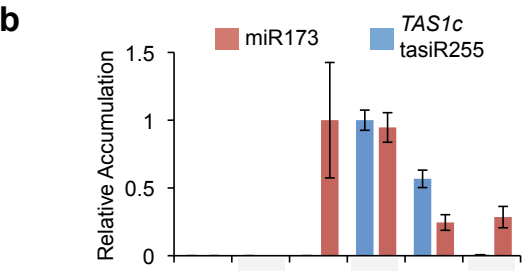

35S:amiR173-21

35S:amiR173

35S:MIR173

Vector

35S:TAS1C

miR173

5 S rRNA/tRNA

SiR255

$5 \mathrm{~S}$ rRNA/tRNA

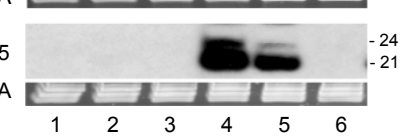

C
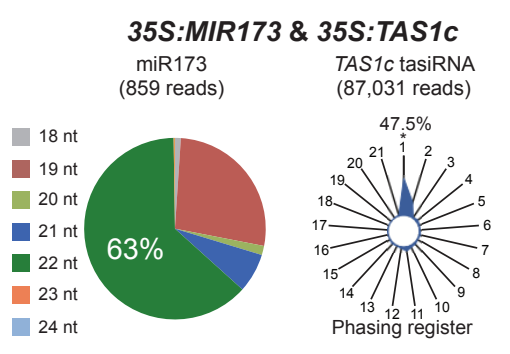

35S:amiR173 \& 35S:TAS1c

miR173

(558 reads)

TAS1C tasiRNA
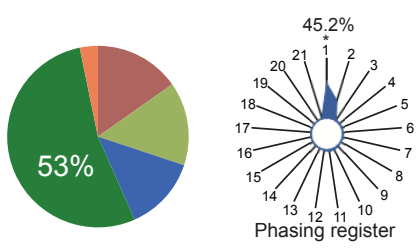

35S:amiR173-21 \& 35S:TAS1c

miR173

(462 reads)

TAS1c tasiRNA

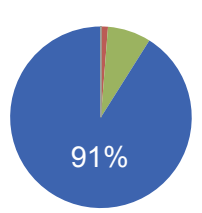

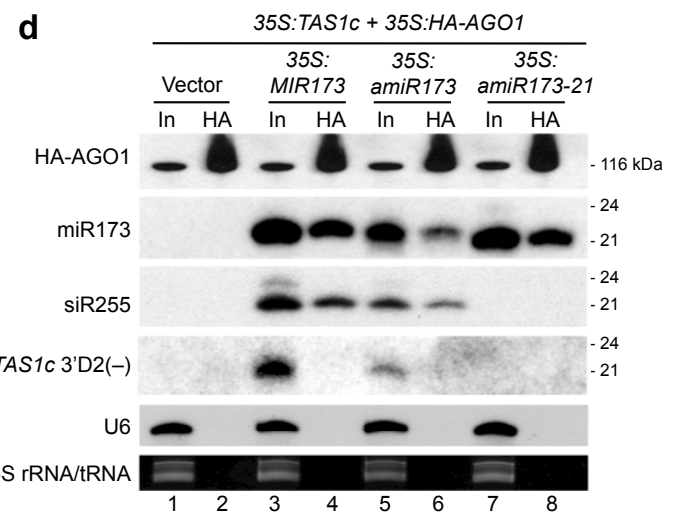

e

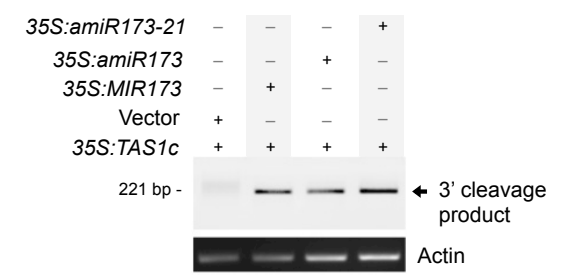

f

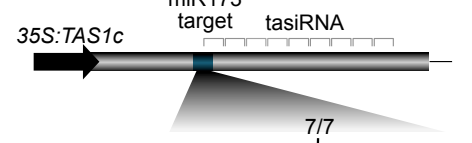

TAS1C 5' GUGAUUUUUCUCUACAAGCGAA 3' amiR173 3' CACUAAAGAGAGACGUUCGCUU 5' TAS1C 5' GUGAUUUUUCUC'UACAAGCGAA 3' amiR173-213, $11|1| 1|1| 1|1| 1|1| 1|1| 1 \mid$

Figure 3 
a

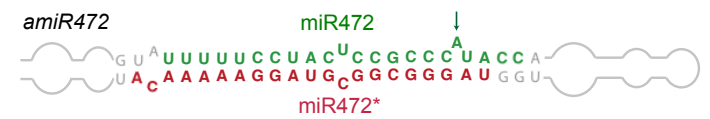

amiR472-21

CUAUUUUUCCUAC CCGCCCAUACA

$\underbrace{\operatorname{miR} 828}_{\text {miR828* }}$

amiR828-21

GUAUCUUGCUUAAAUGGUAUUCCA

C

TAS1c-based constructs

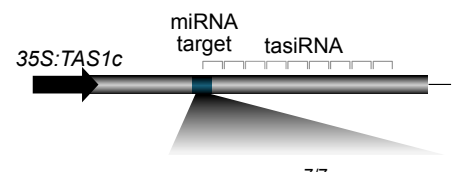

$\stackrel{7 / 7}{\downarrow}$

At1g12290 target 5' GGCAUGGGUGGAGUAGGAAAAA 3 II IIIII: IIIIIIIIIIIIII amiR472 3' CCAUACCCGCCUCAUCCUUUUU 5

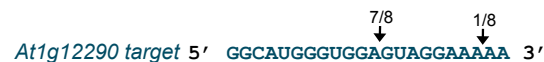
I 111111 1।1111111111111 amiR472-21 $3^{\prime}$ CAUACCCGCCUCAUCCUUUUU $5^{\prime}$ $\stackrel{8 / 8}{t}$

TAS4 target 5' UGGAAUACUCAƯUUGAGCAAGA 3' $111111111111111: 1111111$

amiR828 3' ACCUUAUGAGUAAAUUCGUUCU 5' $7 / 7$

TAS4 target 5' UGGAAUACUCAUUUUGAGCAAGA 3' 11111111111111 :1111111 amiR828-21 3' CCUUAUGAGUAAAUUCGUUCU 5'

d b

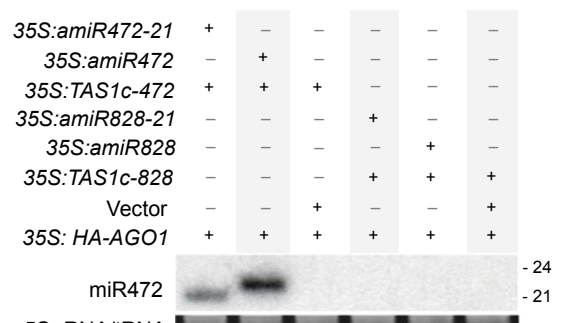

$5 S$ rRNA/tRNA

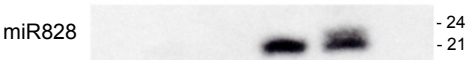

5S rRNA/tRNA $\mathrm{a}=\mathrm{B}$

siR255

$5 \mathrm{~S}$ rRNA/tRNA

$\begin{aligned} & \text { 3' cleavage } \\ & \text { product }\end{aligned}-\longrightarrow-221 \mathrm{bp}$

Actin

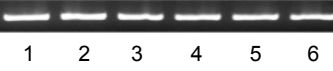

35S:HA-AGO1

\begin{tabular}{|c|c|}
\hline 35S:TAS1c-472 & 35S:TAS1c-828 \\
\hline 35S: & 35S: \\
\hline
\end{tabular}
amiR472-21 amiR472 Vector amiR828-21 amiR828 Vector

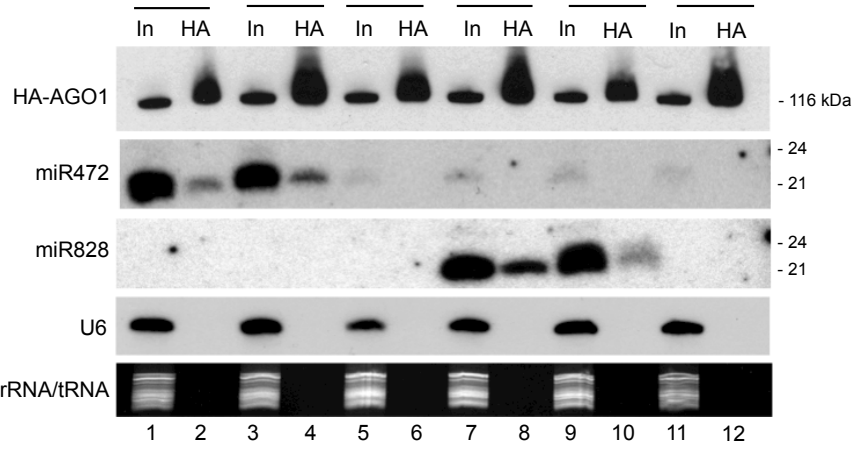

\section{Figure 4}


Supplementary Information for

\section{Unique Functionality of 22 nt miRNAs in Triggering RDR6-Dependent siRNA} Biogenesis from Target Transcripts

By Josh T. Cuperus, Alberto Carbonell, Noah Fahlgren, Hernan Garcia-Ruiz, Russell T. Burke, Atsushi Takeda, Christopher M. Sullivan, Sunny D. Gilbert, Taiowa A. Montgomery and James C. Carrington

\section{SUPPLEMENTAL METHODS}

Small RNA datasets. Small RNA data sets used in this paper are available from Gene Expression Omnibus (GEO, http://www.ncbi.nlm.nih.gov/geo). Data for Arabidopsis wild type Col-0 and $d c / 234$ were previously described ${ }^{1}$ and are available from GEO (GSE20197). Samples used in this work were GSM506656, GSM506657, GSM506658, GSM506662, GSM506663 and GSM506664 for Col-0; and GSM506680, GSM506681, GSM506686, GSM506687 and GSM506688 for $d c / 234$. Data from rice are available from GEO series GSE11014 (GSM278532, GSM278533, GSM278534, GSM278535, GSM278571 and GSM278572)르. GSE16350 (GSM409313, GSM409314, GSM409315, GSM409316, GSM409317, GSM409318, GSM409319, GSM409320, GSM409321, GSM409322, GSM409323 and GSM40924) ${ }^{3}$, GSE16248 (GSM407071 and $\mathrm{GSM}_{407072}{ }^{3}, \mathrm{GSE} 12317\left(\mathrm{GSM} 309691, \mathrm{GSM} 309692\right.$ and GSM309693) ${ }^{4}$, GSE19602 (GSM489087) $^{5}$, and GSE13152 (GSM329296, GSM329297, GSM329298 and GSM329299) 6 .

MIRNA secondary and tertiary structure prediction. MIRNA secondary structures were generated using MC-Fold, a knowledge-based folding algorithm that utilizes data from the Protein Data Bank (PDB) to take into account both canonical Watson-Crick and noncanonical RNA base pairs commonly found in solved RNA structures ${ }^{7}$. 
MIRNA tertiary structures were predicted using MC-Sym ${ }^{7}$. MC-Sym structures were constrained using the MC-Fold structure. Initially, 1000 tertiary models are returned. These initial models were sorted based on all-atoms force-fields and the ten models with lowest free energy were kept. Because MC-Sym constructs tertiary models using fragments from the PDB, the phosphate backbone has stretches of discontinuity. To correct for discontinuity, the models were first relieved until the atomic force's root-meansquare (RMS) was $<100 \mathrm{Kcal} / \mathrm{mol} / \AA$, then refined until the RMS was $<5 \mathrm{Kcal} / \mathrm{mol} / \AA^{7}$. Tertiary structures were viewed using open-source PyMOL v1.2 ${ }^{8}$.

\section{SUPPLEMENTARY REFERENCES}

1. Garcia-Ruiz, H. et al. Arabidopsis RNA-dependent RNA polymerases and dicerlike proteins in antiviral defense and small interfering RNA biogenesis during Turnip Mosaic Virus infection. Plant Cell 22, 481-96 (2010).

2. Zhu, Q.H. et al. A diverse set of microRNAs and microRNA-like small RNAs in developing rice grains. Genome Res 18, 1456-65 (2008).

3. Johnson, C. et al. Clusters and superclusters of phased small RNAs in the developing inflorescence of rice. Genome Res 19, 1429-40 (2009).

4. Zhou, X., Sunkar, R., Jin, H., Zhu, J.K. \& Zhang, W. Genome-wide identification and analysis of small RNAs originated from natural antisense transcripts in Oryza sativa. Genome Res 19, 70-8 (2009).

5. He, G. et al. Global epigenetic and transcriptional trends among two rice subspecies and their reciprocal hybrids. Plant Cell 22, 17-33 (2010).

6. Heisel, S.E. et al. Characterization of unique small RNA populations from rice grain. PLoS One 3, e2871 (2008).

7. Parisien, M. \& Major, F. The MC-Fold and MC-Sym pipeline infers RNA structure from sequence data. Nature 452, 51-5 (2008).

8. DeLano, W.L. The PyMOL molecular graphics system. 1.2 edn (DeLano Scientific LLC, Palo Alto, CA USA, 2008).

9. Rajagopalan, R. et al. A diverse and evolutionarily fluid set of microRNAs in Arabidopsis thaliana. Genes Dev. 20, 3407-3425 (2006). 


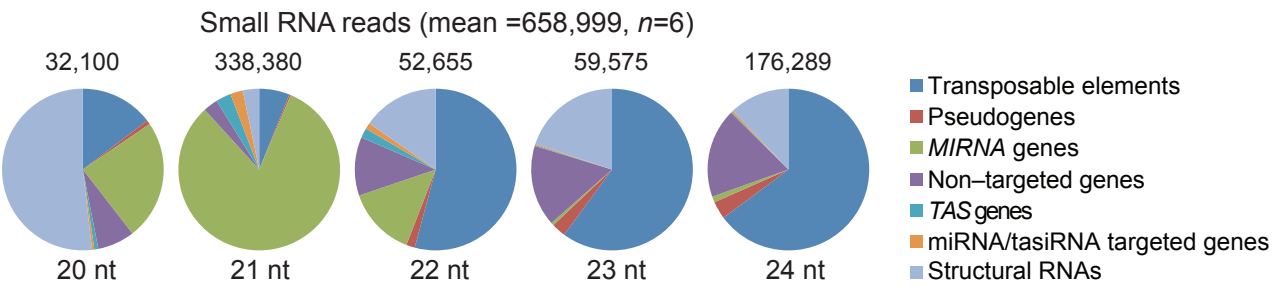

Supplementary Figure 1 Distribution of variously sized small RNA to the indicated features across the Arabidopsis genome. Data are from the means from six libraries. 

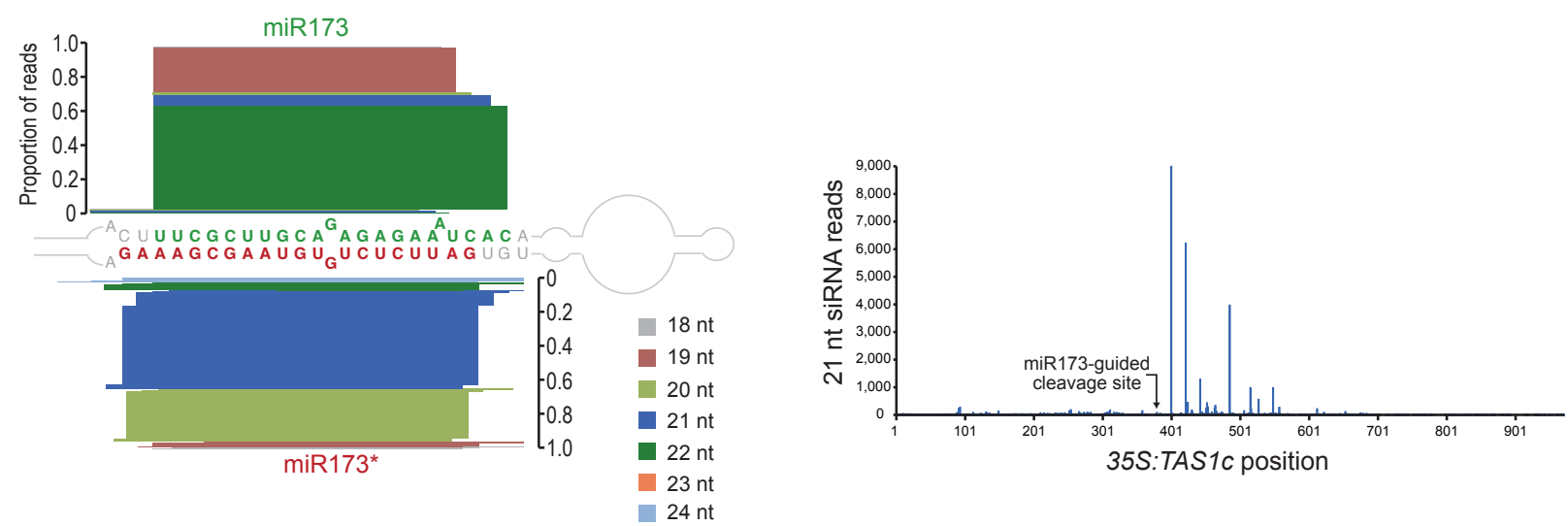

b 35S:amiR173 \& 35S:TAS1c-derived reads
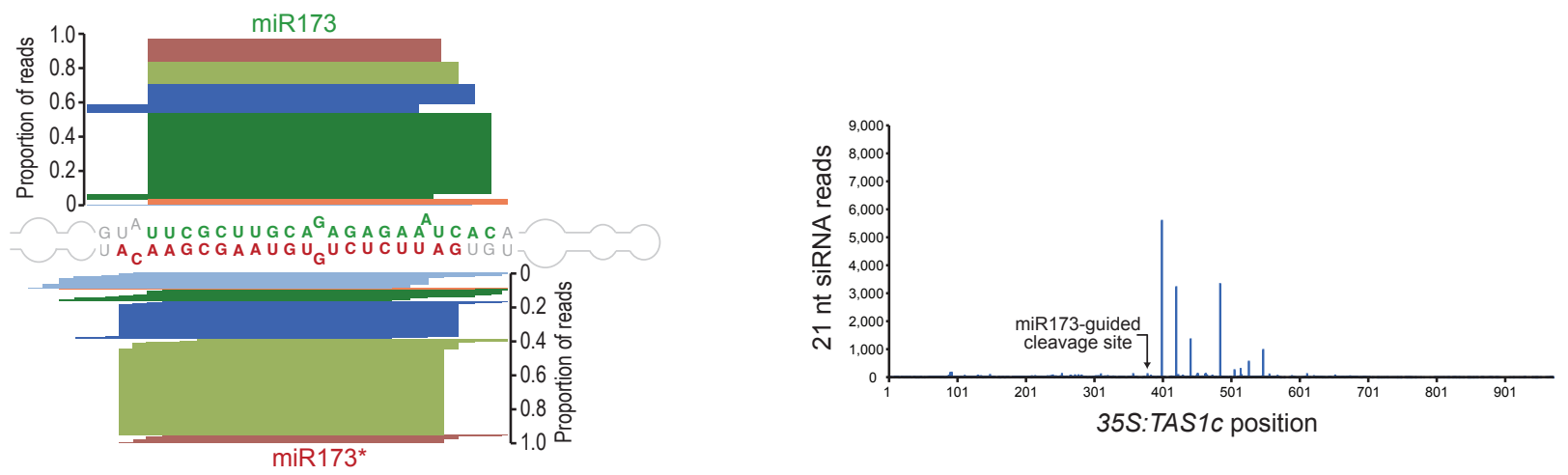

C 35S:amiR173-21 \& 35S:TAS1c-derived reads
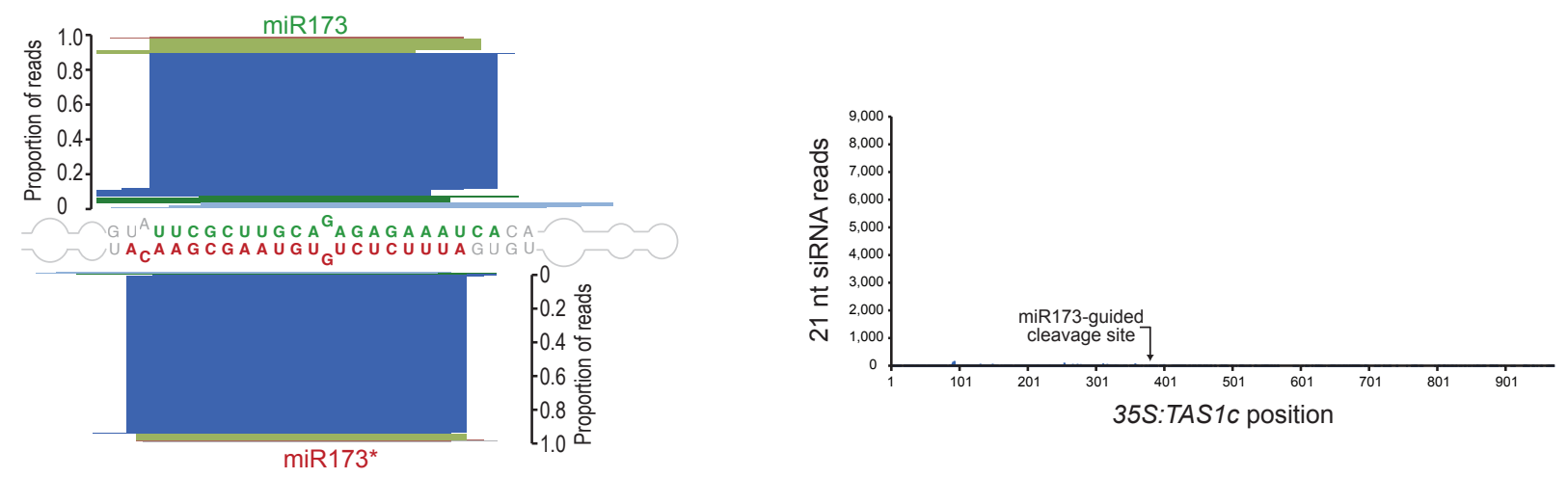

Supplementary Figure 2 Mapping of miR173 or miR173-related reads, and TAS1c transcript-derived reads, from the transient expression assay data shown in Fig. 3c. (a,b,c) Analysis of miR173, miR173* and TAS1c reads after transient co-expression of 35S:MIR173a, 35S:amiR173 or 35S:amiR173-21 with 35S:TAS1c. Proportions of reads are plotted as stacked bars based on size (color coded), 5' position and $3^{\prime}$ position, with end positions aligned to the respective sequences shown in the foldbacks. miR173 and related sequences are plotted upwards, and miR173* sequences are plotted downwards. Twentyone-nucleotide TAS1C siRNA reads are plotted in the bar graphs along the transcript length, with the miR173-guided cleavage site indicated. The bar graphs show consolidated "sense" and "antisense" reads, in which the siRNAs from the two strands were normalized by 2 nts to account for the known offset in duplexes resulting from DCL4-mediated cleavage. 

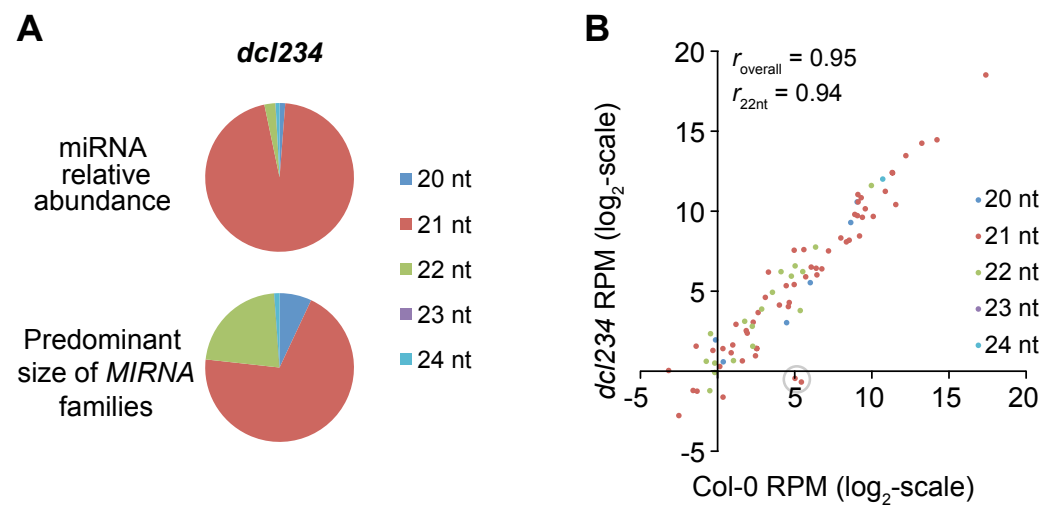

Supplementary Figure 3 miRNA accumulation in the Arabidopsis dc/234 triple mutant. (a) Mean proportions of distinct miRNA size classes in read datasets (top), and of predominant size class for MIRNA families (bottom), from Arabidopsis $d c / 234$ triple mutants. (b) Scatterplot comparing average miRNA reads in wild-type Arabidopsis (Col-0) and dc/234 triple mutants (six and five replicates, respectively). Reads were normalized per million (RPM). The Pearson product-moment correlation coefficients $(r)$ for the overall and $22 \mathrm{nt}$ sets of miRNA families are shown. DCL4-dependent miR822 and miR839 are indicated (grey circle) ${ }^{9}$. 


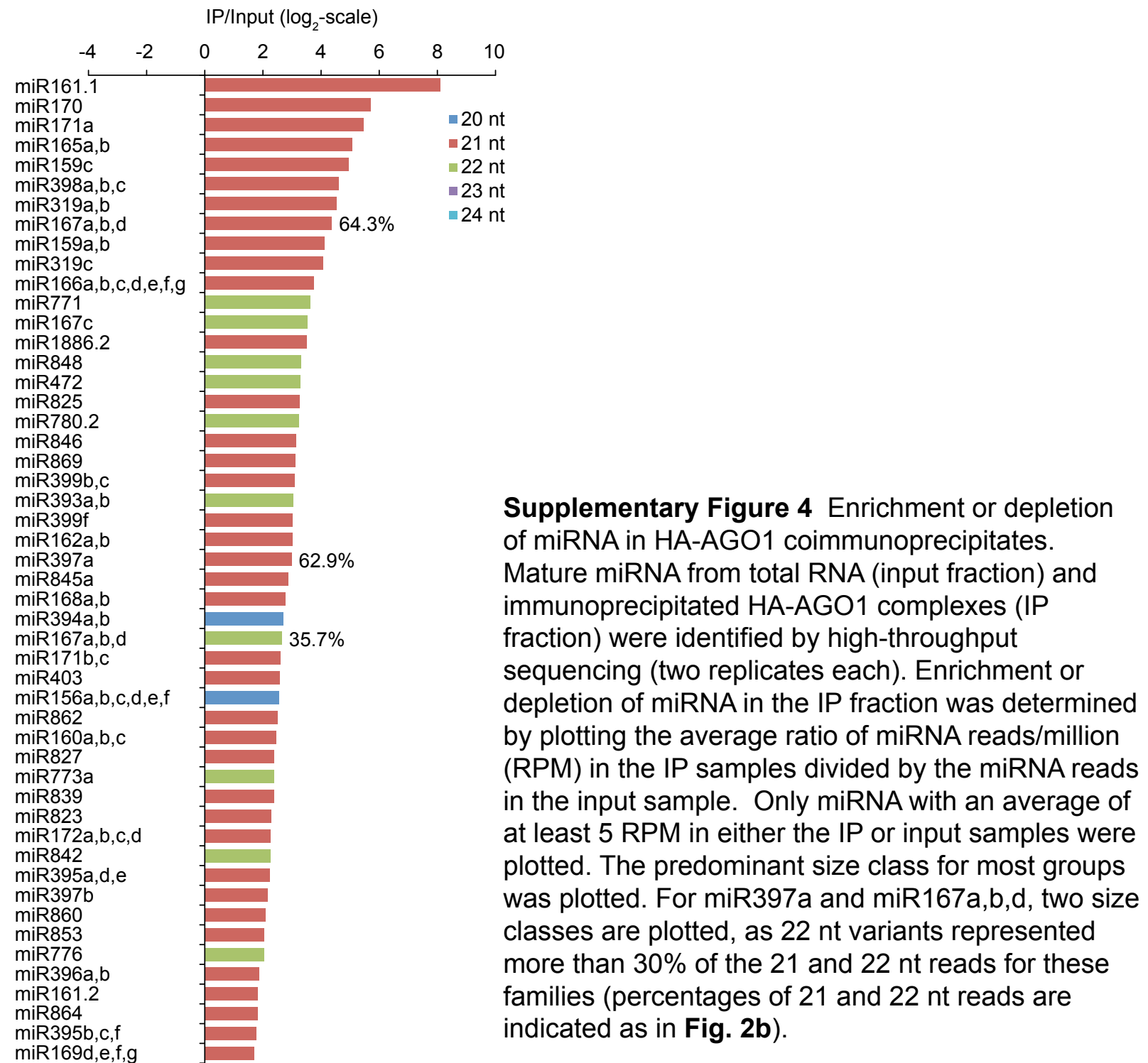




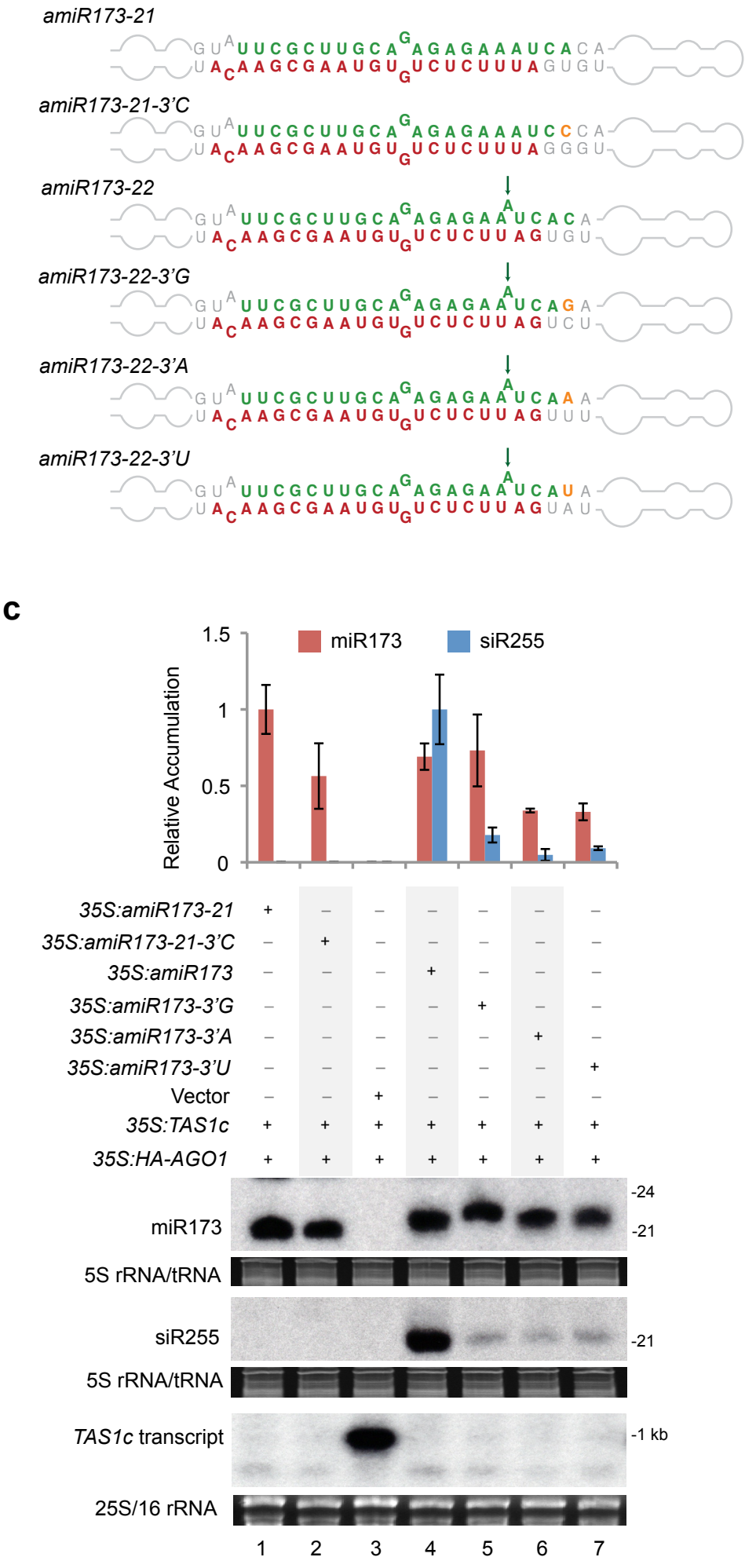

Supplementary Figure 5 Role of the 3' end nucleotide in miR173. (a) Foldbacks yielding $21 \mathrm{nt}$ or $22 \mathrm{nt}$ miR173 variants with distinct 3' end nucleotides. Mutagenized positions are shown in orange. (b) 5'RACE assays showing proportion of products mapping to the canonical cleavage site for each miR173 variant tested. (c) Accumulation of miR173 variants and TAS1c tasiRNA (siR255) in N. benthamiana co-expression assays with the constructs indicated above the blot panels. Mean $(n=3)$ relative miR173 variant (red) and siR255 (blue) levels +/- SD (lane 1 and lane $4=1.0$ for miR173 variant and siR255, respectively). Based on RNA migration, amiR173-22-3'G may be 23 nts in size rather than $22 \mathrm{nts}$. 
a

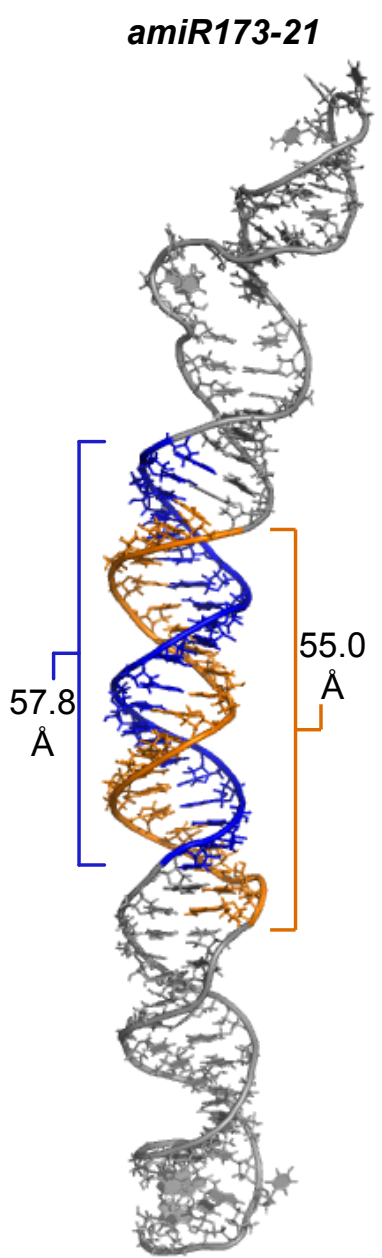

amiR173

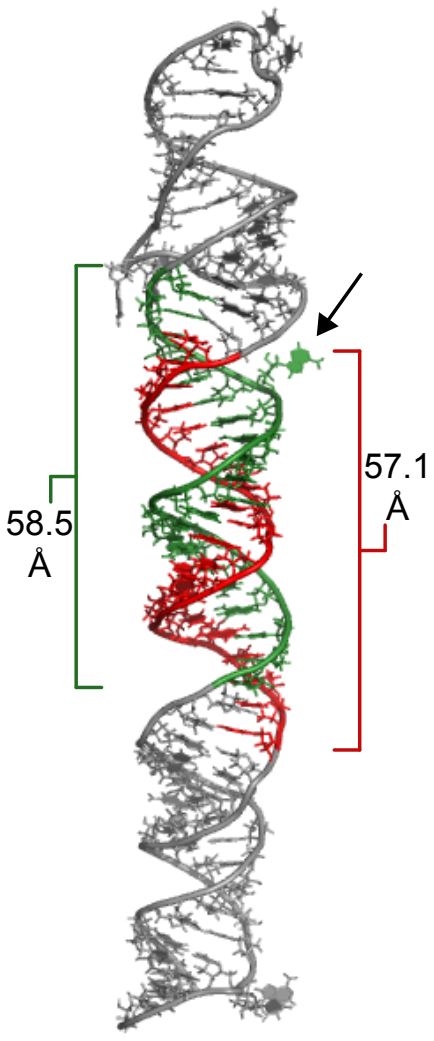

b

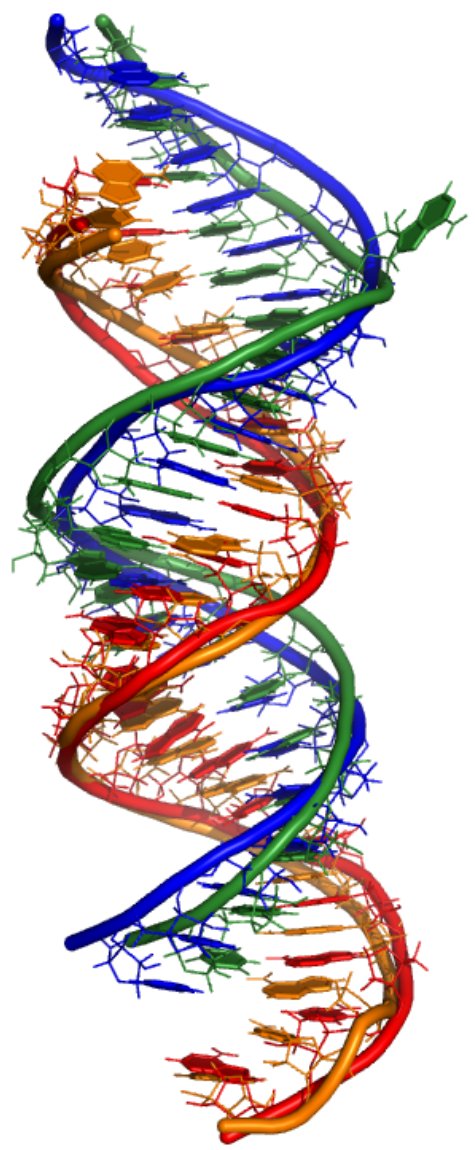

Supplementary Figure 6 Predicted tertiary structures of MIR390a-derived amiR173-21 and amiR173 foldbacks using MC-Fold and MC-Sym. (a) Structures of foldbacks were predicted using MC-Fold and MC-Sym ${ }^{7}$. The amiR173-21 guide and star strands are highlighted blue and orange, respectively. The amiR173 guide and star strands are highlighted green and red, respectively. The position of the nucleotide of the asymmetric bulge is indicated with an arrow. The lengths (in angstroms) of the guide and star strands are shown. (b) Overlay of miRNA/miRNA* duplexes of amiR173-21 and amiR173 show similarity in calculated duplex lengths. 